\title{
Top Physics at the electron proton colliders
}

\author{
Hao Sun* \\ †Institute of Theoretical Physics, School of Physics, \\ Dalian University of Technology, \\ No.2 Linggong Road, Dalian, Liaoning, 116024, P.R.China \\ E-mail: haosun@dlut.edu.cn, haosun@mail.ustc.edu.cn
}

In this talk we present some top physics program at future electron proton colliders. Mentioned topics involve but not limited to top structure function, top parton distribution functions, top spin polarization, top electric charge, measurement of $\mathrm{V}_{\mathrm{tb}}, \mathrm{V}_{\mathrm{ts}}, \mathrm{V}_{\mathrm{td}} \mathrm{CKM}$ matrix elements, anomalous $\mathrm{tt} \gamma, \mathrm{ttZ}$, tbW, tq $\gamma$, tuh couplings and CP phase of tth coupling. The study of flavor changing neutral current tuh couplings and the measurement of $\mathrm{V}_{\mathrm{td}(\mathrm{s})} \mathrm{CKM}$ matrix elements are highlight to show current progress in the top sector.

XXVI International Workshop on Deep-Inelastic Scattering and Related Subjects (DIS2018) 16-20 April 2018

Kobe, Japan

\footnotetext{
* Speaker.

${ }^{\dagger}$ for the LHeC/FCC-eh top physics Study Group
} 


\section{Introduction}

Electron-proton (ep) colliders are hybrids between electron-position $\left(\mathrm{e}^{+} \mathrm{e}^{-}\right)$and proton-proton (pp) colliders, which consist of a hadron beam with an electron beam. They provide a cleaner environment compared to the pp colliders and higher center-of-mass (c.m.s.) energies to the $\mathrm{e}^{+} \mathrm{e}^{-}$ ones. The scenarios studied here involve an electron beam energy of $60 \mathrm{GeV}$ and a LHC proton beam of $7 \mathrm{TeV}(\mathrm{LHeC})$ leading to a c.m.s. energy of $1.3 \mathrm{TeV}$, and a FCC-hh proton beam of 50 $\mathrm{TeV}$ (FCC-eh) leading to a c.m.s. energy of $3.5 \mathrm{TeV}$, respectively. Both projects can deliver up to 2 $\mathrm{ab}^{-1}$ integrated luminosity over their lifetime. Such colliders would become top factories allowing to analyze the electroweak(EW) interactions of the top quark particularly well, and would allow to perform sensitive searches for new physics up to $\mathrm{TeV}$ scale.

This talk focuses on top physics program at the LHeC and FCC-eh which are summarized and quickly mentioned in Section.2. Two selected topics, the study of flavor changing neutral current (FCNC) tuH couplings and the measurement of $\mathrm{V}_{\mathrm{td}(\mathrm{s})} \mathrm{CKM}$ matrix elements are highlight to show current progress in the top sector. Finally, we end this talk with a short summary.

\section{Top quark production at the ep colliders}

By ep collisions, top quark can be produced in pair or singly, through both neutral current (NC) and charged current(CC) productions. The NC top productions include deep inelastic scattering (DIS) or photoproduction $(\gamma \mathrm{p})$ modes, while the $\mathrm{CC}$ ones are through $\mathrm{W}$ boson exchange. Some production rates depending on the collision energy are list in Fig.1. We see that standard

\begin{tabular}{|c|c|c|c|c|c|c|c|}
\hline \multirow[b]{4}{*}{$30 \mathrm{GeV}$} & \multirow[b]{4}{*}{$7 \mathrm{TeV}$} & \multicolumn{3}{|c|}{ top pair } & \multicolumn{3}{|c|}{ single top } \\
\hline & & \multicolumn{2}{|c|}{ NC } & \multirow{2}{*}{$\begin{array}{c}\text { CC } \\
\text { w-exch. }\end{array}$} & \multicolumn{2}{|c|}{ NC } & \multirow{2}{*}{$\begin{array}{c}\text { CC } \\
\text { w-exch. }\end{array}$} \\
\hline & & DIS & rp & & DIS & rp & \\
\hline & & 0. 0040pb & $0.0091 \mathrm{pb}$ & & 4. $653 \mathrm{fb}$ & $12.54 \mathrm{fb}$ & o. $7599 \mathrm{pb}$ \\
\hline $40 \mathrm{GeV}$ & $7 \mathrm{TeV}$ & 0. 0090pb & $0.0205 \mathrm{pb}$ & & 9. $193 \mathrm{fb}$ & 24. $16 \mathrm{fb}$ & 1. $1850 \mathrm{pb}$ \\
\hline $50 \mathrm{GeV}$ & $7 \mathrm{TeV}$ & $0.0165 \mathrm{pb}$ & $0.0354 \mathrm{pb}$ & & 14. $85 \mathrm{fb}$ & $38.27 \mathrm{fb}$ & 1. $6270 \mathrm{pb}$ \\
\hline $60 \mathrm{GeV}$ & $7 \mathrm{TeV}$ & $0.0253 \mathrm{pb}$ & $0.0531 \mathrm{pb}$ & & $21.37 \mathrm{fb}$ & $54.31 \mathrm{fb}$ & 2. $0835 \mathrm{pb}$ \\
\hline $60 \mathrm{GeV}$ & $50 \mathrm{TeV}$ & $0.6268 \mathrm{pb}$ & 1. $1660 \mathrm{pb}$ & & $40.29 f b$ & $942.8 \mathrm{fb}$ & 16. $701 \mathrm{pb}$ \\
\hline
\end{tabular}

Figure 1: Top production rate at ep colliders.

model(SM) top quark production at a future ep collider is dominated by single top quark production, mainly via CC mode. The total cross section is $\sim 2 \mathrm{pb}$ at the $\mathrm{LHeC}$ and $\sim 16 \mathrm{pb}$ at the FCC-eh. The other important top quark production mode is $\bar{t} \bar{t}$ photoproduction with a total cross section of $53 \mathrm{fb}$ at the $\mathrm{LHeC}$ and $\sim 1 \mathrm{pb}$ at the FCC-eh. This makes a future ep collider an ideal tool to study in particular the EW interactions of the top quark.

\section{Selected topics in top sector at the ep colliders}

\subsection{Topics in top sector summarize}

DIS fermion pair production is sensitive to the gluon density in proton. By using DIS $t \bar{t}$ production one can study the top component of the structure function at small $\mathrm{x}$ region[1]. Top 
pair photoproduction can be used to measure the tt $\gamma$ vertex, thus the electric charge of the top quark. When the anomalous $\mathrm{tt} \gamma$ vertex is introduced, top pair photoproduction can also be used to set constraints on the Magnetic and Electric Dipole moment[2]. Similarly but through DIS top pair production from Gluon-Z fusion, one can study the anomalous $t \mathrm{Z}$ couplings, though with less sensitivity. For the anomalous tq $\gamma$ interaction, it is found that ep based $\gamma p$ collider can provide a nice place to probe it[3]. With $1 \mathrm{ab}^{-1}$ integrated luminosity $\mathrm{Br}(\mathrm{t} \rightarrow \mathrm{q} \gamma)$ can be probed up to order of $10^{-6}$, which shows quite nice feature[4].

The CC single top production can be precisely measured through $\mathrm{W}$ boson leptonic or hadronic decay modes, and can be used to measure the CKM matric element $\mathrm{V}_{\mathrm{tb}}$. It is found that at the $\mathrm{LHeC}$, with luminosity of $100 \mathrm{fb}^{-1}, \mathrm{~V}_{\mathrm{tb}}$ can be measured with a precision of $0.5 \%$. In addition, the top quark spin polarization can also be precisely measured through this production[5]. Furthermore, the CC single top production is also a good way to measure the anomalous tbW couplings. Study was performed in [6] by means of the following effective CP conserving lagrangian:

$$
\mathscr{L}_{\mathrm{Wtb}}=\frac{\mathrm{g}}{\sqrt{2}}\left[\mathrm{~W}_{\mu} \overline{\mathrm{t}} \gamma^{\mu}\left(\mathrm{V}_{\mathrm{tb}} \mathrm{f}_{1}^{\mathrm{L}} \mathrm{P}_{\mathrm{L}}+\mathrm{f}_{1}^{\mathrm{R}} \mathrm{P}_{\mathrm{R}}\right) \mathrm{b}-\frac{1}{2 \mathrm{~m}_{\mathrm{W}}} \mathrm{W}_{\mu \nu} \overline{\mathrm{t}} \sigma^{\mu \nu}\left(\mathrm{f}_{2}^{\mathrm{L}} \mathrm{P}_{\mathrm{L}}+\mathrm{f}_{2}^{\mathrm{R}} \mathrm{P}_{\mathrm{R}}\right) \mathrm{b}\right]+\text { h.c. }
$$

where $\mathrm{f}_{1}^{\mathrm{L}}\left(\equiv 1+\Delta \mathrm{f}_{1}^{\mathrm{L}}\right)$ and $\mathrm{f}_{1}^{\mathrm{R}}$ are left- and right-handed vector couplings, $\mathrm{f}_{2}^{\mathrm{L}, \mathrm{R}}$ are left- and righthanded tensor couplings. The analysis shows that the tbW vertex can be probed at the $\mathrm{LHeC}$ to a very high accuracy. There is another CC top production mode making it possible to consider quark density for the top quark, since at very high scales the top may be considered "light". A six-flavor scheme has been proposed in [4], thus the LHeC offers new field of research for the top quark pdfs. The CP-nature of the ttH coupling can also be studied through top-Higgs associated production[7]. The CP-phase dependent lagrangian can be written here,

$$
\mathscr{L}=-\mathrm{i} \frac{\mathrm{m}_{\mathrm{t}}}{\mathrm{v}} \mathrm{t}\left[\kappa \cos \zeta_{\mathrm{t}}+\mathrm{i} \gamma_{5} \sin \zeta_{\mathrm{t}}\right] \mathrm{tH}
$$

Here $\zeta_{\mathrm{t}}$ is the phases of the top-Higgs couplings. The case $\kappa=1, \zeta_{\mathrm{t}}=0$ corresponds to the SM. $\zeta_{\mathrm{t}}=0$ or $\zeta_{\mathrm{t}}=\pi$ correspond to a pure scalar state while $\zeta_{\mathrm{t}}=\pi / 2$ to a pure pseudo scalar state. The ranges $0<\zeta_{\mathrm{t}}<\pi / 2$ or $\pi / 2<\zeta_{\mathrm{t}}<\pi$ represent a mixture of the different CP-states. The study was performed by considering $\mathrm{H}$ to $\mathrm{b} \overline{\mathrm{b}}$ and top quark leptonic decay modes. It was found the LHeC provides a better environment to test the CP nature of Higgs boson couplings compare to LHC.

\subsection{Progress in top sector: $V_{t s}$ and $V_{t d}$ CKM matrix elements}

The signals that involve at least one $\mathrm{V}_{\mathrm{tx}}$ vertex we studied are

$$
\begin{array}{ll}
\text { Signal.1 }: & \mathrm{pe}^{-} \rightarrow v_{\mathrm{e}} \overline{\mathrm{t}} \rightarrow v_{\mathrm{e}} \mathrm{W}^{-} \overline{\mathrm{b}} \rightarrow v_{\mathrm{e}} \ell^{-} v_{\ell} \overline{\mathrm{b}}, \\
\text { Signal.2 }: & \mathrm{pe}^{-} \rightarrow v_{\mathrm{e}} \mathrm{W}^{-} \mathrm{b} \rightarrow v_{\mathrm{e}} \ell^{-} v_{\ell} \mathrm{b}, \\
\text { Signal.3 }: & \mathrm{pe}^{-} \rightarrow v_{\mathrm{e}} \overline{\mathrm{t}} \rightarrow v_{\mathrm{e}} \mathrm{W}^{-} \mathrm{j} \rightarrow v_{\mathrm{e}} \ell^{-} v_{\ell} \mathrm{j}, \\
\text { Signal.4 }: & \mathrm{pe}^{-} \rightarrow v_{\mathrm{e}} \mathrm{W}^{-} \mathrm{j} \rightarrow v_{\mathrm{e}} \ell^{-} v_{\ell} \mathrm{j} .
\end{array}
$$

We present the measurement potential by using different channels separately, depending on the possibility to distinguish them kinematically. But the same final state contributions are fully considered when considering different channels. Our conclusion show that Signal.1, 2 and 3 show very 


\begin{tabular}{|c|c|c|c|c|c|c|c|c|c|}
\hline $\mathscr{P}_{\mathrm{e}}=0.8$ & $\mathrm{LHeC}$ & \multicolumn{2}{|c|}{$1 \mathrm{ab}^{-1}$} & \multicolumn{2}{|c|}{$2 \mathrm{ab}^{-1}$} & \multicolumn{2}{c|}{$1 \mathrm{ab}^{-1}$} & \multicolumn{2}{c|}{$2 \mathrm{ab}^{-1}$} \\
\cline { 3 - 10 } 5\% syst. & $\mathrm{SS}$ & $\mathrm{R}_{\mathrm{d}}$ & $\mathrm{V}_{\mathrm{td}}$ & $\mathrm{R}_{\mathrm{d}}$ & $\mathrm{V}_{\mathrm{td}}$ & $\mathrm{R}_{\mathrm{s}}$ & $\mathrm{V}_{\mathrm{ts}}$ & $\mathrm{R}_{\mathrm{s}}$ & $\mathrm{V}_{\mathrm{ts}}$ \\
\hline \multirow{4}{*}{ signal.1 } & $2 \sigma$ & 8.16 & 0.0699 & 6.86 & 0.0588 & 2.39 & 0.0980 & 2.01 & 0.0824 \\
& $3 \sigma$ & 9.99 & 0.0857 & 8.40 & 0.0720 & 2.92 & 0.1200 & 2.46 & 0.1009 \\
& $5 \sigma$ & 12.90 & 0.1106 & 10.84 & 0.0930 & 3.77 & 0.1550 & 3.17 & 0.1303 \\
\hline \multirow{5}{*}{ signal.2 } & $2 \sigma$ & 10.53 & 0.0903 & 8.85 & 0.0759 & 5.33 & 0.2192 & 4.49 & 0.1843 \\
& $3 \sigma$ & 12.90 & 0.1106 & 10.84 & 0.0930 & 6.53 & 0.2684 & 5.49 & 0.2257 \\
& $5 \sigma$ & 16.65 & 0.1428 & 14.00 & 0.1201 & 8.44 & 0.3465 & 7.09 & 0.2914 \\
\hline \multirow{4}{*}{ signal.3 } & $2 \sigma$ & 6.90 & 0.0592 & 5.83 & 0.0500 & 1.56 & 0.0641 & 1.32 & 0.0541 \\
& $3 \sigma$ & 8.43 & 0.0723 & 7.13 & 0.0611 & 1.91 & 0.0804 & 1.61 & 0.0662 \\
& $5 \sigma$ & 10.82 & 0.0928 & 9.16 & 0.0785 & 2.45 & 0.1008 & 2.07 & 0.0852 \\
\hline
\end{tabular}

Table 1: The 2, 3, and $5 \sigma$ limits for $\mathrm{V}_{\mathrm{td}}$ and $\mathrm{V}_{\mathrm{ts}}$ at the LHeC.

\begin{tabular}{|c|c|c|c|c|c|c|c|c|c|}
\hline $\mathscr{P}_{\mathrm{e}}=0.8$ & FCC-eh & \multicolumn{2}{|c|}{$1 \mathrm{ab}^{-1}$} & \multicolumn{2}{|c|}{$2 \mathrm{ab}^{-1}$} & \multicolumn{2}{|c|}{$1 \mathrm{ab}^{-1}$} & \multicolumn{2}{|c|}{$2 \mathrm{ab}^{-1}$} \\
\cline { 3 - 10 } 5\% syst. & $\mathrm{SS}$ & $\mathrm{R}_{\mathrm{d}}$ & $\mathrm{V}_{\mathrm{td}}$ & $\mathrm{R}_{\mathrm{d}}$ & $\mathrm{V}_{\mathrm{td}}$ & $\mathrm{R}_{\mathrm{s}}$ & $\mathrm{V}_{\mathrm{ts}}$ & $\mathrm{R}_{\mathrm{s}}$ & $\mathrm{V}_{\mathrm{ts}}$ \\
\hline \multirow{4}{*}{ signal.1 } & $2 \sigma$ & 5.46 & 0.0468 & 4.60 & 0.0394 & 1.74 & 0.0716 & 1.47 & 0.0602 \\
& $3 \sigma$ & 6.69 & 0.0573 & 5.63 & 0.0483 & 2.13 & 0.0876 & 1.80 & 0.0738 \\
& $5 \sigma$ & 8.63 & 0.0740 & 7.27 & 0.0623 & 2.75 & 0.1131 & 2.32 & 0.0952 \\
\hline \multirow{5}{*}{ signal.2 } & $2 \sigma$ & 6.46 & 0.0554 & 5.44 & 0.0466 & 2.75 & 0.1131 & 2.32 & 0.0952 \\
& $3 \sigma$ & 7.91 & 0.0679 & 6.66 & 0.0571 & 3.37 & 0.1386 & 2.84 & 0.1166 \\
& $5 \sigma$ & 10.22 & 0.0876 & 8.60 & 0.0737 & 4.35 & 0.1789 & 3.67 & 0.1506 \\
\hline \multirow{4}{*}{ signal.3 } & $2 \sigma$ & 5.01 & 0.0430 & 4.27 & 0.0366 & 1.05 & 0.0433 & 0.90 & 0.0369 \\
& $3 \sigma$ & 6.13 & 0.0526 & 5.22 & 0.0448 & 1.29 & 0.0530 & 1.10 & 0.0452 \\
& $5 \sigma$ & 7.90 & 0.0677 & 6.73 & 0.0577 & 1.66 & 0.0684 & 1.42 & 0.0582 \\
\hline
\end{tabular}

Table 2: The 2, 3, and $5 \sigma$ limits for $\mathrm{V}_{\mathrm{td}}$ and $\mathrm{V}_{\mathrm{ts}}$ at the FCC-eh.

good potential for the measurement of both $\mathrm{V}_{\mathrm{td}}$ and $\mathrm{V}_{\mathrm{ts}}$, and Signal.3 is the most prompt one. In contrary, Signal.4 is of lower quality to be recommended. We displayed the 2,3 , and $5 \sigma$ limits in Tab. 1 for $\mathrm{LHeC}$ and 2 for FCC-eh. We find that considering the proposed $2 \mathrm{ab}^{-1}$ luminosity and $80 \%$ electron polarization, the $2 \sigma$ limits for $\mathrm{R}_{\mathrm{td}}$ parameters for Signal.1, 2, 3 are 6.86, 8.85, 5.83 at the $\mathrm{LHeC}$ and 4.6, 5.44, 4.27 at the FCC-eh, corresponding to the limits of $\mathrm{V}_{\mathrm{td}}$ equal 0.0588 , $0.0759,0.05$ at the LHeC and 0.0394, 0.0466, 0.0366 at the FCC-eh. Similarly, for $\mathrm{R}_{\mathrm{ts}}$, the $2 \sigma \mathrm{R}$ parameter limits are 2.01, 4.49, 1.32 at the $\mathrm{LHeC}$ and 1.47, 2.32, 0.90 at the FCC-eh, corresponding to the limits of $\mathrm{V}_{\text {ts }}$ equal $0.0824,0.1843,0.0541$ at the $\mathrm{LHeC}$ and $0.0602,0.0952,0.0369$ at the FCC-eh. In summary, the electron proton collider provide a nice platform in measuring the $V_{t d}$ and $\mathrm{V}_{\mathrm{ts}} \mathrm{CKM}$ matrix elements.

\subsection{Progress in top sector: FCNC tuH couplings}

We consider two signal productions that contain the top-Higgs FCNC couplings[8]. One is

$$
\text { signal.I }: \mathrm{e}^{-} \mathrm{p} \rightarrow v_{\mathrm{e}} \overline{\mathrm{t}} \rightarrow v_{\mathrm{e}} \mathrm{h} \overline{\mathrm{q}} \rightarrow v_{\mathrm{e}} \mathrm{b} \overline{\mathrm{b}} \overline{\mathrm{q}}
$$


where $\mathrm{q}=\mathrm{u}$ or $\mathrm{c}$. In this case, the five flavor scheme should be applied and an initial state bottom quark will collide with a w boson to produce a single top, which decay anomaly to a Higgs and a light quark. The studied topology gives rise to the $\mathrm{E}_{\mathrm{T}}^{\mathrm{miss}}+$ jets signature characterized by three(or more than three) jets and a missing transverse momentum( $\left(\mathrm{E}_{\mathrm{T}}^{\mathrm{miss}}\right)$ from the undetected neutrino. Two of the jets should be tagged as B-jets. The combination of the two B-jets should appear as a narrow resonance centered around the SM Higgs boson mass. Together with the remaining light jet(s), they should be able to reconstruct a resonant top quark. The second channel we considered is

$$
\text { singal.II }: \mathrm{e}^{-} \mathrm{p} \rightarrow v_{\mathrm{e}} \mathrm{hb} \rightarrow v_{\mathrm{e}} \mathrm{b} \overline{\mathrm{b}} \mathrm{b}
$$

where the FCNC tqh couplings are induced through light quarks that directly emitting from the proton. Considering the studied topology, three tagged B-jets should be required for signal.II. The Feynman diagrams are plotted in Fig.2.

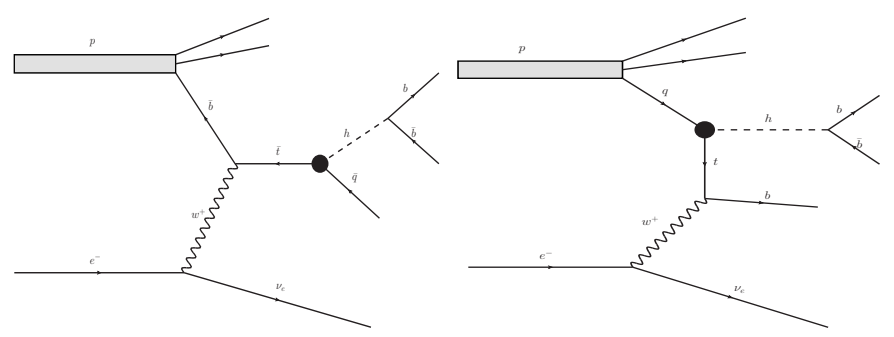

Figure 2: Illustrated Feynman diagrams for the processes $\mathrm{e}^{-} \mathrm{p} \rightarrow v_{\mathrm{e}} \overline{\mathrm{t}} \rightarrow v_{\mathrm{e}} \mathrm{h} \overline{\mathrm{q}} \rightarrow v_{\mathrm{e}} \mathrm{b} \overline{\mathrm{b}} \overline{\mathrm{q}}$ (signal.I) and $\mathrm{e}^{-} \mathrm{p} \rightarrow v_{\mathrm{e}} \mathrm{hb} \rightarrow v_{\mathrm{e}} \mathrm{b} \overline{\mathrm{b}} \mathrm{b}$ (signal.II) at the ep colliders that contain flavor changing top-Higgs interactions.

In Fig.3, the upper limit on $\mathrm{Br}(\mathrm{t} \rightarrow \mathrm{uh})$ at $99.99,99.73,95.40,68.27 \%$ C.L. as a function of the integrated luminosity are plotted. Our conclusion is that, for signal.I, at the high luminosity (up to $1 \mathrm{ab}^{-1}$ ) ep colliders where the electrons have a polarisation of $80 \%$, the $1 \sigma, 2 \sigma, 3 \sigma$ and $5 \sigma$ upper limit on $\operatorname{Br}(\mathrm{t} \rightarrow \mathrm{uh})$ are $0.075 \times 10^{-2}\left(0.14 \times 10^{-3}\right), 0.15 \times 10^{-2}\left(0.29 \times 10^{-3}\right), 0.22 \times 10^{-2}(0.43 \times$ $\left.10^{-3}\right)$ and $0.38 \times 10^{-2}\left(0.72 \times 10^{-3}\right)$ at the $\mathrm{LHeC}(\mathrm{FCC}-\mathrm{eh})$. For signal.II, the boundaries are becoming $0.064 \times 10^{-2}\left(0.097 \times 10^{-3}\right), 0.15 \times 10^{-2}\left(0.22 \times 10^{-3}\right), 0.26 \times 10^{-2}\left(0.35 \times 10^{-3}\right)$ and $0.53 \times 10^{-2}\left(0.68 \times 10^{-3}\right)$ at the $\mathrm{LHeC}(\mathrm{FCC}$-eh $)$ respectively. We can see that signal.II can even have better potential than signal.I at the FCC-eh due to its clean environment. Notice here we use $5 \%$ systematic uncertainty for backgrounds yields only at both ep colliders.

\section{SUMMARY}

In this talk we present an overview of top physics at the ep colliders. Mentioned topics involve but not limited to top structure function, top parton distribution functions, top spin polarization, top electric charge, measurement of $\mathrm{V}_{\mathrm{tb}}, \mathrm{V}_{\mathrm{ts}}, \mathrm{V}_{\mathrm{td}} \mathrm{CKM}$ matrix elements, anomalous $\mathrm{tt} \gamma, \mathrm{tt}$, tbW, tq $\gamma$, tuh couplings and $\mathrm{CP}$ phase of th coupling, etc. The flavor changing neutral current tuh couplings, and the study of $\mathrm{V}_{\mathrm{td}(\mathrm{s})} \mathrm{CKM}$ matrix elements are highlight to show current progress. New ideas or contributions are also welcomed in order to further emphasize the strength of the LHeC (FCC-eh) when it comes to the top sector. 

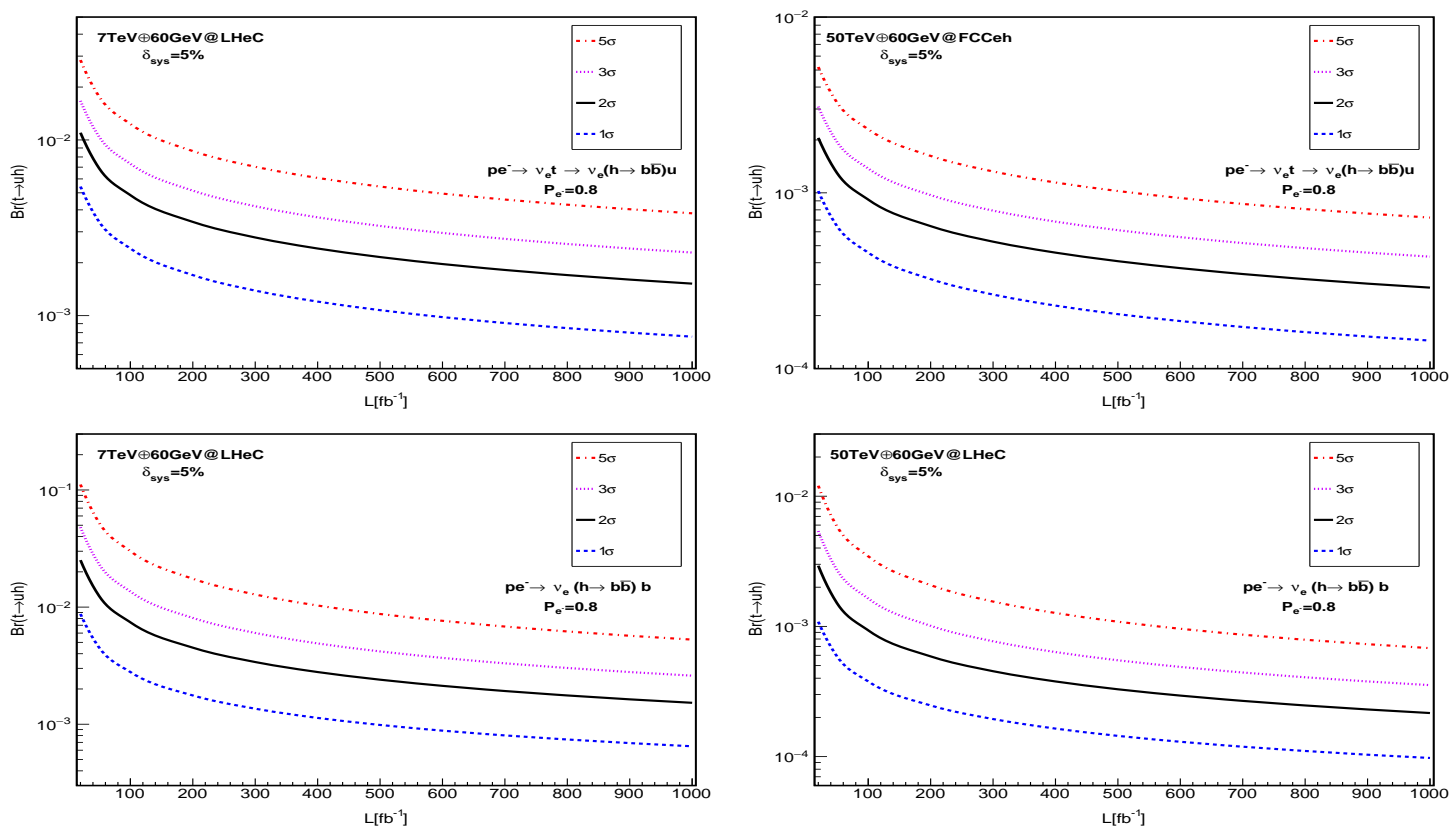

Figure 3: The upper limit on $\operatorname{Br}(\mathrm{t} \rightarrow \mathrm{uh})$ at $99.99,99.73,95.40,68.27 \%$ C.L. as a function of the integrated luminosity at the $\mathrm{LHeC}(\mathrm{FCC}-\mathrm{eh})$.

\section{References}

[1] G.R.Boroun, Geometrical scaling behavior of the top structure functions ratio at the LHeC, Phys.Lett.B744 (2015) 142-145; Top structure function at the LHeC, Phys.Lett.B741 (2015) 197-201.

[2] A.O.Bouzas and F.Larios, Probing tt $\gamma$ and ttZ couplings at the LHeC, Phys.Rev.D 88 (2013) 094007, [arXiv: 1308.5634$]$.

[3] I.T.Cakir, O.Cakir and S.Sultansoy, Anomalous Single Top Production at the LHeC Based $\gamma p$ Collider, Phys.Lett.B 685 (2010) 170-173, [arXiv: 0911 . 4194].

[4] LHeC Study Group, A Large Hadron Electron Collider at CERN: Report on the Physics and Design Concepts for Machine and Detector, J.Phys.G 39 (2012) 075001, [arXiv : 1206 . 2913].

[5] S.Atag and B.D.Sahin, Top quark spin in ep collision, Phys.Rev.D 69 (2004) 034016.

[6] S.Dutta, A.Goyal, M.Kumar and B.Mellado, Measuring anomalous W tb couplings at $e^{-}$p collider, Eur.Phys.J.C 7512 (2015) 577, [arXiv:1307.1688].

[7] Baradhwaj Coleppa, Mukesh Kumar, Satendra Kumar and Bruce Mellado, Measuring CP nature of top-Higgs couplings at the future Large Hadron electron Collider, Phys.Lett.B 770 (2017) 335-341, [arXiv:1702.03426].

[8] Hao Sun, Xuan Luo, Jing Li, Exploring the Anomalous Top-Higgs FCNC Couplings at the electron proton colliders, Eur.Phys.J.C 78 (2018) no.4, 281, [arXiv: 1602.04670 ]; XiaoJuan Wang, Hao Sun and Xuan Luo, Searches for the Anomalous FCNC Top-Higgs Couplings with Polarized Electron Beam at the LHeC, Adv.High Energy Phys 2017 (2017) 4693213, [arXiv: 1703 . 02691]; Wei Liu, Hao Sun, XiaoJuan Wang, Xuan Luo, Probing the anomalous FCNC top-Higgs Yukawa couplings at the Large Hadron Electron Collider, Phys.Rev.D 92 (2015) no.7, 074015, [arXiv: 1507.03264 ]. 